Instituto Internacional de Investigación y Desarrollo Tecnológico Educativo INDTEC, C.A.

DOI: https://doi.org/10.29394/Scientific.issn.2542-2987.2020.5.15.13.269-287

OAI-PMH: http://www.indteca.com/ojs/index.php/Revista Scientific/oai

Artículo Original / Original Article

\title{
Mirada Fenomenológica desde las Competencias Investigativas en las Líneas de Investigación de la UNESR
}

\author{
Autora: Yolanda del Carmen Díaz de Salas \\ Universidad Nacional Experimental "Rafael María Baralt", UNERMB \\ ydiazdesalas@yahoo.es \\ Trujillo, Venezuela \\ http://orcid.org/0000-0002-4785-2396
}

\section{Resumen}

La praxis del investigador, refleja su pensamiento y un hacer forjado en instituciones educativas que han aportado perspectivas culturales, basadas en experiencias individuales y grupales, producto de un compartir en grupo académico, contribuyente al proceso de adquisición de Competencias Investigativas. De allí, el propósito general del artículo es analizar la metodología fenomenológica relacionada a las Competencias Investigativas que desarrolla el estudiante universitario en las Líneas de Investigación de la Universidad Nacional Experimental "Simón Rodríguez" (UNESR). Metodológicamente, se corresponde con el Paradigma Interpretativo, el recorrido metodológico fenomenológico utilizado, fue el modelo de Rodríguez, Gil y García (1996). Los actores clave del estudio, fueron cuatro (4) egresadas de la Maestría Ciencias de la Educación de Educación Avanzada, que desarrollaron su investigación en cuatro (4) líneas de Investigación de la UNESR, Núcleo Valera. Es decir, 1. Fortalecimiento de la Educación Inicial (LinFEI); 2. Investigadores de Lengua y Literatura (ILLA); 3. Gerencia y Tecnología (LinGERTEC); y 4. Educación y Pobreza (CEDIEPO). La información se recolectó por medio de la técnica: Entrevistas en Profundidad, en total fueron doce (12) entrevistas. Como resultado, fueron tres (3) categorías para generar las Competencias Investigativas Integradas tales como son: Competencia del Saber Conocer; Competencia del Saber Ser y Competencia del Saber Hacer.

Palabras clave: metodología; investigación; educación universitaria.

Cómo citar este artículo:

Díaz de Salas, Y. (2020). Mirada Fenomenológica desde las Competencias Investigativas en las Líneas de Investigación de la UNESR. Revista Scientific, 5(15), 269-287, e-ISSN: 2542-2987. Recuperado de: https://doi.org/10.29394/Scientific.issn.2542-2987.2020.5.15.13.269-287

Fecha de Recepción: 19-06-2019
Fecha de Aceptación:

04-10-2019
Fecha de Publicación: 05-02-2020 


\title{
Phenomenological view from the Research Competencies in the Research Lines of the UNESR
}

\begin{abstract}
The praxis of the researcher, reflects his thinking and a work forged in educational institutions that have contributed cultural perspectives, based on individual and group experiences, product of an academic group sharing, contributing to the process of acquisition of Research Competencies. From there, the general purpose of the article is to analyze the phenomenological methodology related to the Research Competencies that the university student develops in the Research Lines of the National Experimental University "Simón Rodríguez" (UNESR). Methodologically, it corresponds to the Interpretive Paradigm, the phenomenological methodological route used, was the model of Rodríguez, Gil and García (1996). The key actors in the study were four (4) graduates of the Master of Science in Advanced Education Education, who developed their research in four (4) lines of research at UNESR, Valera Nucleus. That is, 1. Strengthening Initial Education (LinFEI); 2. Language and Literature Researchers (ILLA); 3. Management and Technology (LinGERTEC); and 4. Education and Poverty (CEDIEPO). The information was collected through the technique: Interviews in Depth, in total there were twelve (12) interviews. As a result, there were three (3) categories to generate the Integrated Investigative Competencies such as: Knowledge Knowledge Competence; Competence of Knowing Being and Competence of Knowing How.
\end{abstract}

Keywords: methodology; research; university education.

\section{How to cite this article:}

Díaz de Salas, Y. (2020). Phenomenological view from the Research Competencies in the Research Lines of the UNESR. Revista Scientific, 5(15), 269-287, e-ISSN: 2542-2987. Recovered from: https://doi.org/10.29394/Scientific.issn.2542-2987.2020.5.15.13.269-287

Date Received: 19-06-2019
Date Acceptance:

04-10-2019
Date Publication: 05-02-2020 


\section{Introducción}

EI Paradigma Interpretativo, explica la naturaleza del artículo mostrado, asimismo, el recorrido metodológico propio del Método Fenomenológico, destellado en gestiones que muestran, cada una de las etapas y pasos que lo caracterizan. Es decir, se presenta la manera de abordar contextos sobre las experiencias, percepciones, expresiones de los actores clave, al igual que los métodos y técnicas del respectivo análisis.

Para realizar estudios en las Ciencias Sociales el Paradigma Interpretativo, según Taylor y Bogdan (1986), mencionado por Rodríguez, Gil y García (1996a), consideran en un sentido amplio, la investigación cualitativa como: "aquella que produce datos descriptivos: las propias palabras de las personas habladas o escritas, y la conducta observable" (pág. 33). Estos autores señalan que es inductiva; las personas, los escenarios, se ven desde una perspectiva holística, son métodos más humanistas, tratan de comprender a las personas dentro del marco de referencia de ellas mismas.

La mayor parte analítica se realiza mediante las voces de los actores clave, para categorizarlas siguiendo procesos cognitivos de agrupar, unir o fraccionar semióticamente. Esto se hace, para permitirle al investigador contraste, compare y analice para así construir la teorización. Los anteriores planteamientos, convergen en las tres actividades que considera Denzin y Lincoln (1994a), citado por Rodríguez, Gil y García (1996b): que definen "el proceso de investigación cualitativa a partir de actividades genéricas interconectadas entre sí: la ontología, epistemología y la metodología" (pág. 41).

Ante el planteamiento ontológico, la concepción del hombre para el estudio, es considerado como el ser que construye y reconstruye en su interacción social, cuya reconstrucción se da desde lo exterior a lo interior. En este sentido, Habermas (1984), señala que: "la ciencia como actividad social está impregnada de los valores e intereses de quienes la desarrollan" (pág. 
113). Por esto, se debe abordar la problemática sin prejuicios y con abreviada disposición a la reflexión dialógica para generar los cambios que se requieren.

Por su parte, la relación sujeto-objeto en el conocimiento (epistemológico) está centrada en que el discernimiento, la subjetividad surgen de y en la interpretación y descripción de los fenómenos entre los sujetos de estudio. Ante estos planteamientos, la epistemología compleja, según Morín (2000): precisa de "un pensamiento complicado que coloque la atención al conocimiento mismo: una vez que se reconoce la cualidad del sujeto como observador-conceptualizador, el conocimiento del conocimiento se situará como punto de partida, complejizando la noción misma de epistemología" (pág. 117).

Es así, como de la ontología y epistemología depende la metodología, la cual permite llegar a la construcción y reconstrucción de significado a partir de los actores, con la aplicación de métodos y técnicas basados en procesos hermenéuticos, diálogos que suceden intentando sacar sentido de sus expresiones desde la interpretación de los significados que tiene, para las personas implicadas. Por lo antes expuesto, se consideró el paradigma interpretativo como la vía que orienta este trabajo, por cuanto, permite comprender la completa y subjetiva realidad.

En este momento, es necesario resaltar la postura filosófica que guio a la investigadora a realizar el estudio, debido a que se requirió de una fundamentación representada por una triada de conocimientos pertinentes entre sí, en la cual se percibió la coherencia que oriento la indagación, con respecto a la ontología o naturaleza del sujeto cognoscente, la epistemología o teoría del conocimiento adecuada y la metodología o forma de formalizar la praxis investigativa.

Respecto, al enfoque de la investigación que guarda sustento con el artículo mostrado, adopta el enfoque cualitativo, porque permite la construcción de conocimiento a partir de las vivencias y sentires de los actores 
clave, por surgir como alternativa para realizar estudios en ciencias sociales cuyo interés es llegar a la comprensión de las realidades inmersas dentro de ámbitos para unificar, porque no podemos buscar variables que dependan o sean independientes.

Así mismo, Pérez (2000), establece que: "el enfoque cualitativo pretende ofrecer profundidad y a la vez el detalle mediante una descripción y registro cuidadoso" (pág. 21). En otras palabras, en el contexto educativo el enfoque cualitativo significa desarrollo, en ese caso en las Ciencias Sociales, debido a que estudian la realidad sociocultural, con todas sus estructuras cuyo fin es buscar comprensiones holísticas.

En ese mismo orden de ideas, Sandín (2003), expresa que: "constituye un concepto amplio que hace referencia a diversas perspectivas epistemológicas, teóricas, incluyendo también numerosos métodos y estrategias de investigación" (pág. 122). Atendiendo a estas consideraciones, se asume como postura epistemológica el enfoque cualitativo, permitiendo llegar a indagar cómo es la relación, que se da en el contexto de los actores objeto de estudio. Por esto, el objeto de estudio de esta Tesis Doctoral que dio origen al artículo en mención, es analizar la metodología fenomenológica relacionada a las Competencias Investigativas que desarrolla el estudiante universitario en las Líneas de Investigación de la Universidad Nacional Experimental "Rafael María Baralt" (UNESR).

Desde esa perspectiva, el término competencias según lo aludido por Tobón (2006), citado por Pérez y Quintero (2016), al señalar que: "las competencias académicas del docente representan un saber hacer o saber actuar en forma responsable, validada en un contexto profesional particular, combinando, recursos necesarios, conocimientos, habilidades, actitudes para lograr un resultado óptimo dentro de su práctica diaria" (pág. 193).

Por tanto, el estudio cumplió con las pautas de las investigaciones cualitativas: porque no se hace posible separar a los sujetos del contexto en 
el que realizan su cotidianidad y actuaciones; el punto de vista, la interpretación y los resultados dependen de la percepción de los actores clave tal cual cómo ellos lo perciben. Para esto, se asumió la Fenomenología como método, explicado con detalles a continuación.

\section{Marco Referencial}

\subsection{Fenomenología}

Según Husserl (1990), citado por Bolio (2012a), respecto al concepto de fenomenología, se refiere a que:

La fenomenología designa un nuevo método descriptivo que hizo su aparición en la filosofía a principios del siglo (siglo xx) y una ciencia apriorística que se desprende de él y que está destinada a subministrar el órgano fundamental para una filosofía rigurosamente científica y posibilitar, en un desarrollo consecuente, una reforma metódica de todas las ciencias (págs. 22-23).

De ahí, que el objeto de la fenomenología es básicamente, descubrir en el fenómeno su esencia para validarla generalmente, y hacerla de utilidad científica. Es decir, para acceder al mundo de la conciencia pura es necesario, partir de actitudes radicales, denominada epojé trascendental.

De este modo, debo abstenerme de mi visión, mis reflexiones, no conceptúo, por lo tanto, la Epojé es fundamental en la fenomenología, así Bolio (2012b), establece que:

Con la epojé fenomenológica el sujeto se gana a mí mismo como subjetividad que, en parte, autodefine su mirar en función de sus proyectos, de sus procesos transformadores. Puedo entonces imaginar e imaginarme más allá del aquí y del ahora. Estoy frente a mí mismo como "subjetividad trascendental" (pág. 25).

En otras palabras, el fenomenólogo habrá de concebir al mundo no desde su percepción, sino desde la subjetividad de la cual forma parte en el 
mundo. En consecuencia, posicionados bajo el enfoque cualitativo, en esta indagación abordé el método fenomenológico el cual desarrollé con base a los señalamientos de Morse (2003), citado en Barbera e Inciarte (2012): exponen "la importancia metodológica de la fenomenología en relación a que son enfoques adecuados dentro de la investigación cualitativa, están centradas en las experiencias vividas, comportamientos, sentimientos, funcionamiento organizacional, entre otros" (pág. 201).

Bajo esta perspectiva, el método fenomenológico de los citados autores, delimita como ámbito para su análisis la conciencia y su intencionalidad a ello, provocando que, en su trayectoria de las "cosas mismas", se pase a un ámbito notable, o lo que es lo mismo, que dicha fenomenología es trascendental. Tal es el caso, de este contexto fundamentado en las competencias investigativas del estudiante de educación avanzada en las Líneas de Investigación de la UNESR, Núcleo Valera.

\section{Metodología}

\subsection{Método Fenomenológico}

La metodología bajo el enfoque cualitativo, con el método fenomenológico, se origina en la antropología que para Goetz y LeCompte (1988): "consiste en una teoría de la descripción; concepción que reafirma la percepción de que la fenomenología es el reflejo de la realidad, producto de la interacción humana" (pág. 112). Para reafirmar lo expuesto por los citados autores, se considera en forma analítica que la investigación fenomenológica es interpretativa, con valor al protagonismo humano para crear interpretaciones significativas en el entorno social y físico. Por ello, en mi inquietud por la investigación, asumí el método fenomenológico.

De acuerdo con lo antes expuesto, tal es el caso de las integrantes egresadas de la Maestría Ciencias de la Educación de la UNESR, quienes aportaron una serie de cualidades en forma de descripciones completas de 
acontecimientos, interacciones y actividades que llevaron a la autora al descubrimiento de categorías y relaciones que ayudaron a la interpretación de dichos datos.

\subsubsection{Recorrido Metodológico}

Consciente de que las investigaciones cualitativas, buscan generar una construcción del conocimiento en colectivo, basado en su realidad cotidiana, requiere de la comprensión de realidades interactivas, captar significados, interpretar el sentido profundo de las acciones de las personas y grupos, hasta llegar a un modo de hacer ciencia de lo social, requiere de un procedimiento epistemológico- metodológico que lo garantice.

De manera que, en el recorrido metodológico utilizado por la investigadora se razona acerca de las fases de esta metodología fenomenológica conocidas y reflexionadas, al pretender abordar la subjetividad del individuo con este método. Entendiendo, que la fenomenología ha de ser imparcial, descriptiva para descubrir lo que de inalterable hay en un fenómeno, captando entonces la esencia de éste. De aquí se parte, para insertar el proceso de comprensión que la investigadora asumió para desplegar acciones específicas a cumplir a través de las fases con sus respectivas acciones y llevar así a feliz término el recorrido fenomenológico.

\subsubsection{Fase Preparatoria}

Esta fase inicial de la investigación cualitativa está constituida por las etapas: reflexiva y diseño. En la etapa reflexiva a juicio de Rodríguez, Gil y García (1996c): "el investigador posiblemente intentará clarificar, determinar el tópico de interés y describir las razones por las que elige el tema. Identifica un

tópico de investigación supone elegir desde qué claves o coordenadas de pensamiento se desea afrontar la comprensión..." (pág. 65). 
En ese orden de ideas, la etapa reflexiva; se evidenció de manera explícita, en consideraciones reflexivas de la autora, su inquietud a investigar, donde se reflexiona sobre la observancia de dificultades y problemáticas con escasa adquisición de competencias para indagar, en las Líneas de Investigación. Aludiendo lo expuesto por Rodríguez, Gil y García (1996d): "como resultado final de esta etapa, el investigador puede disponer del marco teórico en el que va a desarrollar su investigación y que va a utilizar como referencia para todo el proceso" (pág. 67).

Luego, en la etapa de Diseño, desde los aportes de Rodríguez, Gil y García (1996e): "se suele especificar el proceso de selección que se va a llevar a cabo para asegurarse que el lugar o las personas objeto de investigación (el escenario) se acerquen lo más posible a lo ideal" (pág. 69). De manera que, la autora consideró necesario la estructuración del estudio, con el propósito rector del mismo, identificación del contexto y los actores clave, método, técnica, interrogantes, fechas; todos estos aspectos representaron la concepción del diseño como un trabajo ordenado, secuenciado, que indica la ontología, epistemología y a la vez, la metodología.

Obviamente, esta fase dependió de la elección paradigmática expuesta en la etapa anterior, por ello el "diseño de la investigación", se planteó a la luz del propósito general, como guía para llegar a la verdad científica, comprender y captar el sentido profundo de acciones que le dan los actores clave, en su propio contexto a través, de las entrevistas en profundidad.

En lo concerniente, a los contextos o escenarios de investigación fueron: cuatro (4) instituciones educativas y la Universidad Nacional Experimental "Simón Rodríguez" (UNESR), Núcleo Valera, por ser entes de formación, donde hacen vida activa todos los entrevistados del proceso educativo. A continuación, se muestran las etapas de la fase preparatoria, en la figura 1. 
Figura 1. Etapas de Fase Preparatoria.

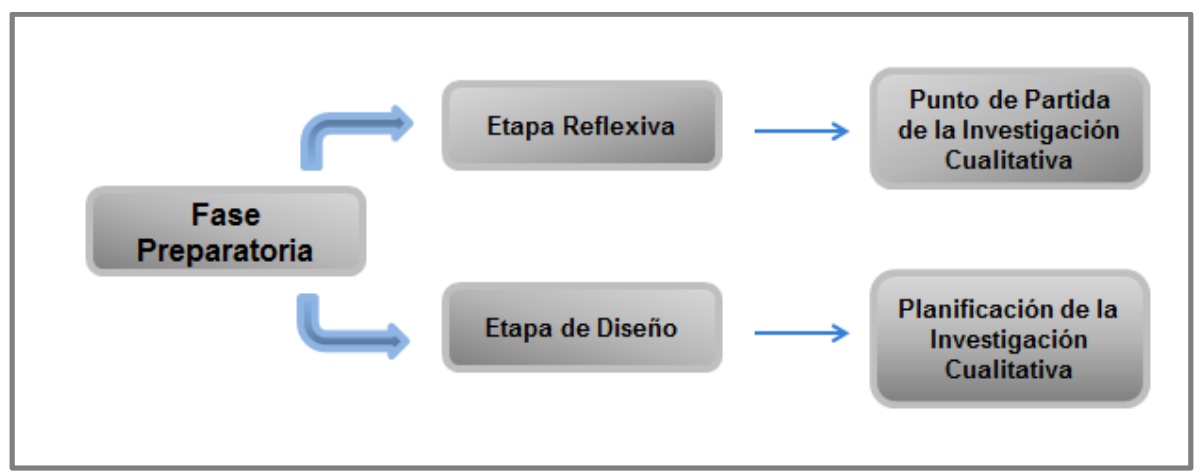

Fuente: La Autora (2018).

\subsubsection{Fase de Trabajo de Campo}

En esta etapa, el investigador demostrará sus destrezas y astucias para entrar al campo de estudio, de manera que se pueda obtener una buena investigación cualitativa. En palabras de Rodríguez, Gil y García (1996f): "si en la fase de preparación había que tener en cuenta la formación y experiencia del investigador en este momento del estudio, resulta de una importancia crucial algunas características del mismo que permitirán el avance de la investigación" (pág. 71). Amparados en esta visión, el investigador se debe enfrentar al cumplir esta fase de la investigación a un acceso al campo para recoger productivamente, la información que servirá para luego analizarla e ir avanzando a la siguiente fase.

Ahora bien, el acceso al campo según, Rodríguez, Gil y García (1996g): "se entiende como un proceso por el que el investigador va accediendo progresivamente a la información fundamental para su estudio" (pág. 72). Es decir, el investigador debe crear ese clima de confianza para que los informantes puedan expresarse sin prejuicios, de forma que su comunicación devele información. Es por ello, que el acceso al campo para este estudio doctoral resultó gratificante debido a que la investigadora logró una afinidad y confianza con los involucrados, conoce su cultura, costumbres, valores, 
principios, ética; lo que permitió el acercamiento resultando de forma cordial, amable y abierta, dispuestos a colaborar con la investigación.

Asimismo, en la Recogida Productiva de Datos en esta fase la Investigadora, se planteó la búsqueda de significados, reflexiones, experiencias y perspectivas de los actores o informantes a la luz de las interrogantes. Así, la entrevista realizada para el estudio doctoral, adoptó la forma de un dialogo coloquial, según Martínez (2006): "la gran relevancia, las posibilidades y la significación del diálogo como método de conocimiento de los seres humanos estriba, sobre todo, en la naturaleza y calidad del proceso en que se apoya" (pág. 93). De manera, que se realizaron doce (12) entrevistas en profundidad por cada interrogante, a los cuatro (4) tipos de actores clave del estudio, las cuales se transcribieron tal como lo expresaron.

Además, durante la entrevista, se llevó un registro descriptivo y grabadora, para ello se solicitó permiso a la entrevistada para grabar la plática. Esto facilitó la confrontación de las anotaciones con la grabación, convirtiéndose en vía expedita de validación de la experiencia vivida con los entrevistados: sus sentimientos, intuiciones, dudas, satisfacciones académicas y personales. A continuación, se muestran las etapas de la fase de trabajo de campo, en la figura 2.

Figura 2. Etapas de Fase de Trabajo de Campo.

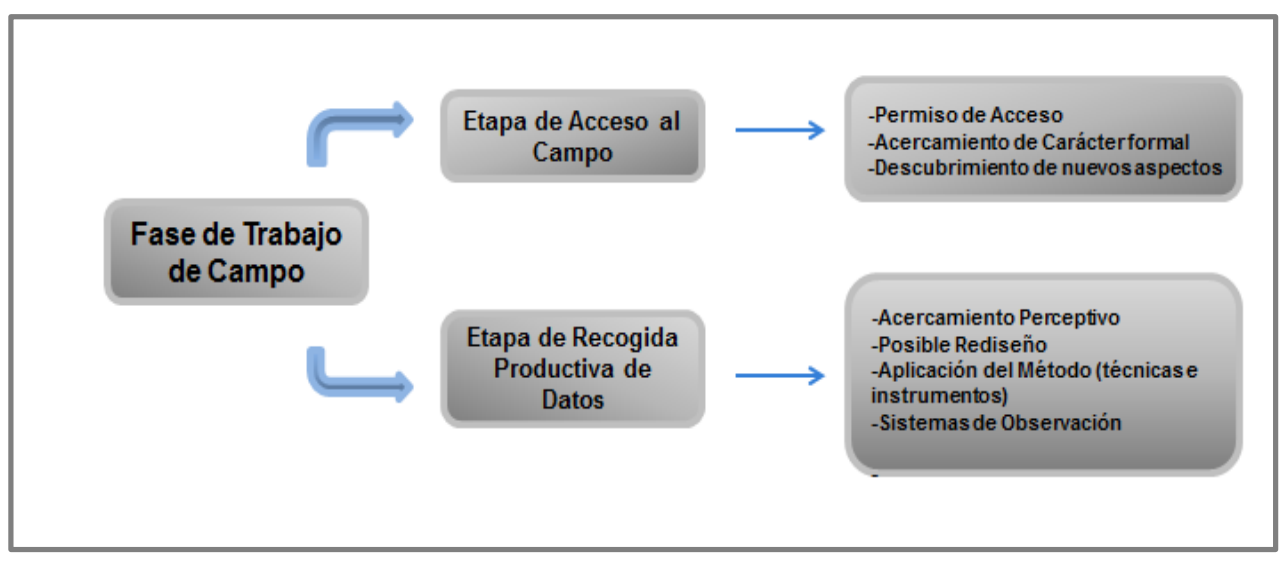

Fuente: La Autora (2018). 


\subsubsection{Fase Analítica}

Para ejecutar esta fase, se acude al proceso cognitivo de analizar la información obtenida, esto se hace al abandonar el investigador, el escenario propio de la indagación. En palabras, de Rodríguez, Gil y García (1996h):

Es considerado como un proceso realizado con cierto grado de sistematización que a veces permanece implícita en las actuaciones emprendidas por el investigador... es posible establecer una serie de tareas u operaciones que constituyen el proceso analítico básico común a la mayoría de los estudios en que se trabaja con datos cualitativos. Estas tareas serían: a). reducción de datos; b). disposición y transformación de datos; y c). obtención de resultados y verificación de conclusiones (pág. 75).

El análisis realizado partió desde la transcripción de dialogo obtenido en las entrevistas en profundidad, de allí se extrajeron las expresiones, términos incluidos relevantes, los cuales, al ser interpretados, resaltan las subcategorías o relaciones semánticas para llegar a los dominios o las denominadas categorías. Sobre la base de las expresiones de los estudiantes implicados en las cuatro (04) líneas de investigación de la UNESR, quienes compartieron espacios de investigación, en pro de la construcción de competencias investigativas. Sus expresiones, opiniones, actividades, sentimientos, las relaciones entre el grupo, se transcribieron luego, fueron interpretadas sus realidades, las cuales se ubicaron en categorías.

En cuanto, a codificar, se traduce en asignarle a cada unidad temática un código adecuado a la categoría que estamos trabajando, para dejar constancia que se realizó la categorización. De manera, que en este estudio se hicieron todas estas tareas de asociación a códigos y categorías, resultantes de todas las expresiones, manifestaciones, actitudes.

Por último, referente al análisis triangular, visto por Denzin y Lincoln (1994b), citado por Pérez (2003): como "el proceso que permite la combinación de metodologías en el estudio de un mismo fenómeno" (pág. 81). Por tanto, 
para este estudio se considerarán una serie de acciones que permitirán chequear la información obtenida utilizando diferentes fuentes de información, con el propósito de contrastar y comparar la diversidad de testimonios.

Se hizo este proceso, una vez que se logró las categorías emergentes en las expresiones captadas en la técnica utilizada, a fin de llegar a la construcción de un todo, así obtener la visión global de los hallazgos. Según el autor citado; existen tres tipos de triangulación en virtud del objeto de estudio: la triangulación metodológica, la temporal o de momentos y la de informantes y sujetos, tomando en cuenta las diversas opiniones, expresiones, juicos de los sujetos implicados en dicho estudio. Luego, se confrontaron los diversos o coincidentes puntos de vistas.

Asimismo, la información recogida permitió establecer una analogía productiva, ingeniosa y creativa. Es decir, se confrontó la información suministrada por éstos, con la postura de autores, sobre el tema y la visión de la investigadora. Esto llevó, a los procesos de validez y fiabilidad de los resultados, dependen en gran medida de la importancia de argumentos desarrollados. En la figura 3, se muestran las etapas que conforman la fase analítica.

Figura 3. Etapas de Fase Analítica.

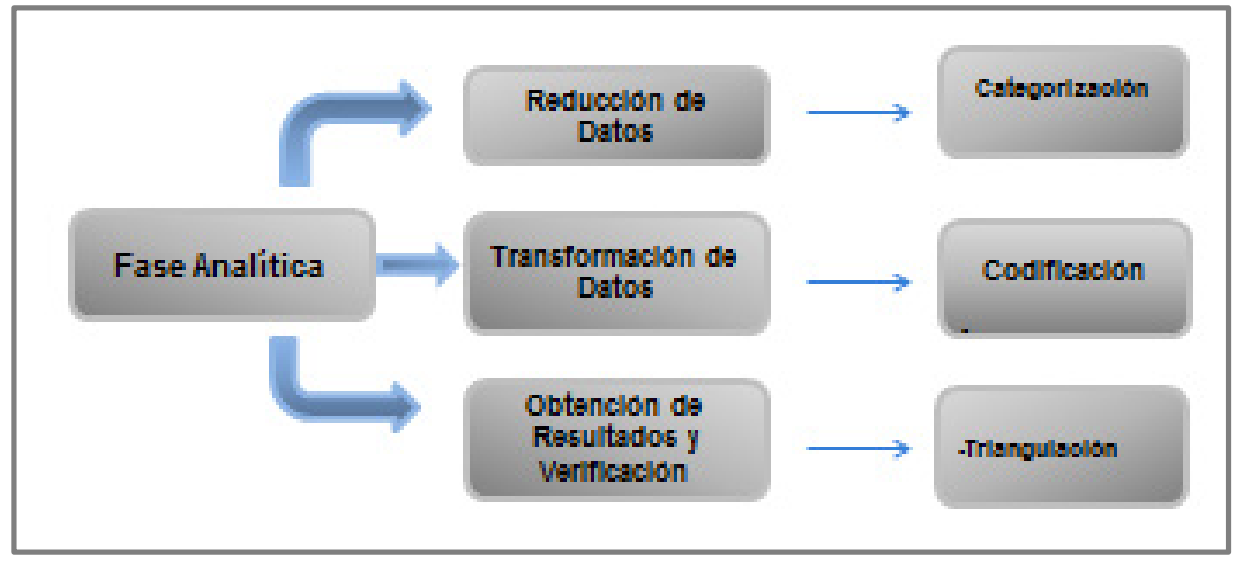

Fuente: La Autora (2018). 
Al respecto, Rodríguez, Gil y García (1996i), señalan que la validez de una investigación: "puede ser definida por el grado o nivel que los resultados de la investigación reflejen una imagen clara y representativa de una realidad o situación dada" (pág. 322); la fiabilidad, según los mismo autores Rodríguez, Gil y García (1996j): "es un estudio se puede repetir con el mismo método sin alterar los resultados de la investigación" (pág. 325).

\subsubsection{Fase Informativa}

Esta fase, e característica del reflejo de culminar, presentar y difundir los resultados. Al respecto, Rodríguez, Gil y García (1996k): manifiesta que "el informe cualitativo debe ser argumento convincente, presentando los datos sistemáticamente, que apoyen el caso del investigador y refute las explicaciones alternativas" (pág. 76). Es necesario recalcar, que a partir del texto elaborado por el investigador, donde integra notas de campo con respectivos documentos, se elaborará el informe de la indagación recreado por la interpretación del investigador sacando a la luz lo aprendido.

A continuación, se muestra la figura 4 , correspondiente a la fase informativa.

Figura 4. Fase Informativa.

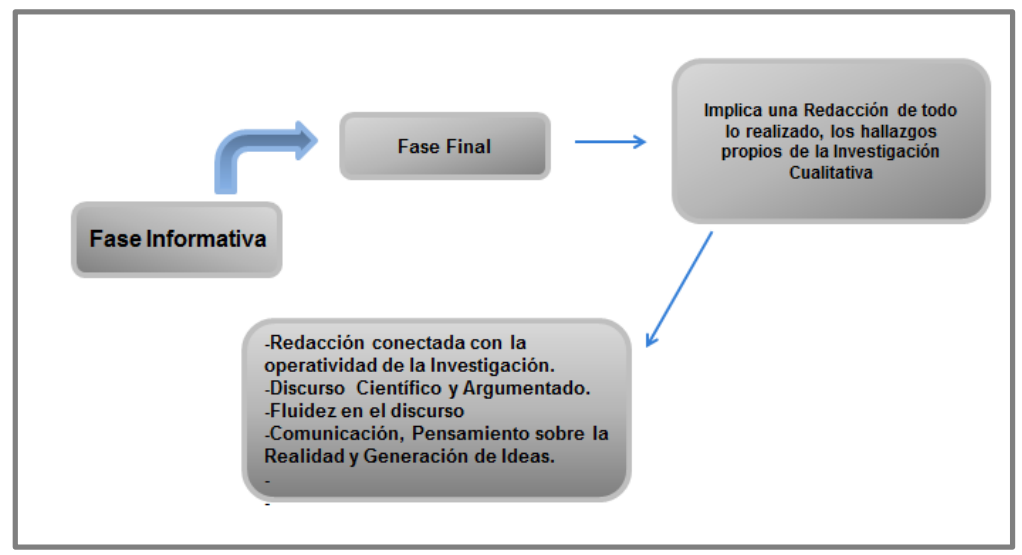

Fuente: La Autora (2018). 


\section{Análisis e interpretación de los resultados}

Para efectos de la validez y confiabilidad en la investigación, la autora procede a triangular las categorías halladas: Competencia del Saber Conocer, Competencia del Saber Ser y Competencia del Saber Hacer, tomando en consideración lo expuesto por Rodríguez, Gil y García (1996I), donde presenta tres (3) tipos de Triangulación:

La Triangulación Metodológica, que se produce en dos (2) direcciones; por un lado, se han conjugado datos de naturaleza cualitativa y cuantitativa, dando lugar a los diseños mixtos y por otro lado, los métodos interpretativos, para estudiar el mismo fenómeno. El otro tipo de triangulación es, la temporal o de momento; que implica hacer uso combinado de métodos longitudinales y transversales, de forma que los primeros implican recoger datos procedentes del mismo grupo en distintos momentos de la secuencia temporal. El tercer tipo de Triangulación es de Informantes y Sujetos; corresponde a Conocer y Contrastar los múltiples puntos de vista que conjugan en una misma circunstancia constituye una práctica recomendable y esencial si se pretende aproximarse a un entendimiento profundo de la realidad que permita interpretaciones justificadas (pág. 311).

Sobre la base de la Triangulación de Informantes y Sujeto, la autora del estudio selecciona este tipo de Triangulación, debido a que buscó indagar en los Integrantes de cuatro diferentes líneas de investigación de la Universidad Nacional Experimental "Simón Rodríguez" (UNESR), a fin de Interpretar las Competencias Investigativas adquiridas en las respectivas líneas.

Una vez concluida la triangulación de datos, los resultados de la información obtenida a través de la técnica Entrevista en Profundidad, realizada con cuatro (4) informantes claves de las cuatro Líneas de Investigación: Fortalecimiento de la Educación Inicial (LinFEI); Investigadores de Lengua y Literatura (ILLA); Gerencia y Tecnología (LinGERTEC); y Educación y Pobreza (CEDIEPO); se determina que existen o favorecen a los estudiantes que ingresan y egresan de las líneas, las tres dimensiones de una 
competencia; el saber conocer, basados en conocimientos factuales y declarativos. El saber hacer, fundamentalmente en habilidad, destreza y procedimientos y el saber ser, el cual está enmarcado en las actitudes y valores.

\section{Consideraciones Finales}

La metodología fenomenológica, se eligió porque para mí representa una experiencia invaluable, adentrar en las vivencias de los sujetos actores de la indagación representa un conocimiento de saberes y experiencias que no tienen comparación. De hecho, en mi acto de investigación viví experiencias agradables, enriquecedoras que marcan mi andar académico. Por esto, en base a cada categoría resultante del estudio planteo las consideraciones:

En cuanto a la Categoría del Saber Conocer: Adquirir competencias investigativas en cualquier contexto, requiere de un proceso de construcción científicos, en el cual, los estudiantes universitarios, desarrollan habilidades cognitivas que permiten; diseñar, plantear y ejecutar los proyectos de investigación. En tal sentido, las Líneas de Investigación son las garantes de dichas fases de Investigación.

Asimismo, con la Categoría del Saber Ser: construir competencias investigativas, involucra variedad de aspectos caracterizados en ese saber ser como persona, con afectividad lo cual es significativo para investigar venciendo todos los obstáculos que se presenten. Aunando a ella, está la ayuda y colaboración de un grupo de otros que actúan como mediadores oportunos, haciendo un ambiente interactivo desde el ser afectivo.

Por último, la categoría del Saber Hacer: fortalecer las Competencias Investigativas requiere de un trabajo cooperativo con una cultura del esfuerzo para formar en el hacer al futuro investigador. Las actividades cónsonas a llegar a una función productiva, se establece en el consenso en la Línea de Investigación. 


\section{Referencias}

Barbera, N., \& Inciarte, A. (2012). Fenomenología y hermenéutica: dos perspectivas para estudiar las ciencias sociales y humanas. Multiciencias, 12(2), 199-205, e-ISSN: 1317-2255. Recuperado de: http://redalyc.org/articulo.oa?id=90424216010

Bolio, A. (2012a,b). Husserl y la fenomenología trascendental: Perspectivas del sujeto en las ciencias del siglo XX. Reencuentro, (65), 20-29, e-ISSN: 0188-168X. Recuperado de: http://www.redalyc.org/articulo.oa?id=34024824004

Denzin, N. \& Lincoln, Y. (1994a,b). The SAGE Handbook of Qualitative Research. First edition, ISBN: 0-7619-2757-3. Thousand Oaks, EE. UU.: SAGE Publishing.

Goetz, J., \& LeCompte, M. (1988a.b). Etnografía y Diseño Cualitativo en Investigación Educativa. ISBN: 978-84-7112-320-6. Madrid, España: Ediciones Morata.

Habermas, J. (1984). El discurso filosófico de la Modernidad. ISBN: 3-51857722-0. Madrid, España: Editorial Taurus.

Husserl, E. (1990). Artículo de la Enciclopedia Británica. Cuarta y última versión, Publicada originalmente en alemán en 1925. México: UNAM.

Martínez, M. (2006). Ciencia y arte en la Metodología Cualitativa. ISBN: 968-24-7011-0. México, D.F.: Editorial Trillas.

Morse, J. (2003). Asuntos críticos en los métodos de investigación cualitativa. Colombia: Editorial Universidad de Antioquia.

Morín, E. (2000). Introducción al pensamiento complejo. ISBN: 978-847432-518-8. Barcelona, España: Gedisa Editorial, S.A.

Pérez, G. (2000). Investigación Cualitativa I: Retos e Interrogantes: Métodos. $6^{\circ}$ Edición, ISBN: 9788471336286 . Madrid, España: Editorial La Muralla, 234 págs.

Pérez, M. (2003). Pedagogía Social. Educación Social. Construcción 
científica e intervención práctica. ISBN: 84-277-1440-8. Madrid, España: Narcea, S.A. de Ediciones.

Pérez, W., \& Quintero, E. (2016). Competencias Docentes para la Construcción del Conocimiento de los Estudiantes de Educación

Primaria. Revista Scientific, 1(1), 190-211, e-ISSN: 2542-2987. Recuperado de:

https://doi.org/10.29394/scientific.issn.2542-2987.2016.1.1.11.190-211

Rodríguez, G., Gil, J., \& García, E. (1996a,b,c,d,e,f,g,h,i,j,k,l). Metodología de la Investigación Cualitativa. ISBN 10: 8487767567; ISBN 13: 9788487767562. Granada, España: Ediciones Aljibe, 380 págs.

Sandín, M. (2003). Investigación Cualitativa en Educación, Fundamentos y Tradiciones. ISBN: 84-481-3779-5. Barcelona, España: McGraw-Hill Interamericana de España, S.L.

Taylor, S., \& Bogdan, R. (1986). Introducción a los Métodos Cualitativos de Investigación. ISBN 10: 8475098169; ISBN 13: 9788475098166. Buenos Aires, Argentina: Paidós Ibérica Ediciones, S.A.

Tobón, S. (2006). Aspectos básicos de la formación basada en Competencias. Talca, México: Proyecto Mesesup. Recuperado de: http://maristas.org.mx/gestion/web/doctos/aspectos basicos formacio $\underline{\text { n competencias.pdf }}$ 


\section{Yolanda del Carmen Díaz de Salas \\ e-mail: ydiazdesalas@yahoo.es}

Nacida en la ciudad de Valera, estado Trujillo, Venezuela,

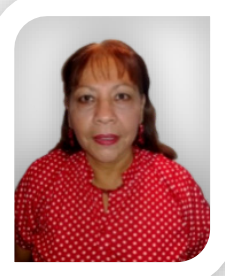

el 21 de noviembre del 1956. Profesional universitaria en

el área de Gerencia de Recursos Humanos; Doctora en Ciencia de la Educación, egresada de la Universidad Nacional Experimental "Rafael María Baralt" (UNERMB); $\mathrm{He}$ participado como Docente Contratada en la Universidad Nacional Experimental Politécnica de la Fuerza Armada (UNEFA), Betijoque, estado Trujillo; Desempeño el cargo de docente de aula, en la Universidad Bolivariana de Venezuela (UBV), Municipio Valera, estado Trujillo. Además, poseo conocimientos avanzados en cuanto al manejo de Windows XP; Microsoft Office (Word, Excel, PowerPoint); Lulo Software; AutoCAD Avanzado 2D e Internet.

El contenido de este manuscrito se difunde bajo una Licencia de Creative Commons ReconocimientoNoComercial-Compartirlgual 4.0 Internacional 